\title{
Illustration of Cowling channel coupling to the shear Alfven wave
}

\author{
A. Yoshikawa ${ }^{1,2}$ \\ ${ }^{1}$ Dept. of Earth and Planetary Sciences, Kyushu University \\ ${ }^{2}$ Space Environment Reserch Center, Kyushu University
}

A complete formulation of Cowling channel coupling to the field-aligned current (FAC) system via shear Alfven wave is developed. The theoretical framework of this formulation does not depend on the specific geometry (like an auroral arc) and can describe the formation process of Cowling channel without using any parameterization of the Cowling effect. Namely, our newly developed formulation can identify how much of the polarization electric field is generated via preventing the Hall current to flow, and how much of the Hall current flow out to the magnetosphere via the FAC.

We introduce a new concept of conjugate current system, in which the Hall effect is defined to act in the opposite direction to that of the actual current system. Similar to the methodology that the real and imaginary parts of a complex number are extracted from addition and subtraction between the complex number and their complex conjugate, the actual current system can be separated into two orthogonal current systems with some algebraic operation between the actual and conjugate current system. The one separated system includes the Hall-Pedersen parallel circuit corresponds to the electrojet current enhanced by the Cowling effect and the second one includes the Hall-Pedersen antiparallel circuit, in which the Hall polarization field is generated. A simple numerical test validates our theoretical framework.

The formulation derived in this manuscript is the first and the complete description of the Cowling channel coupling to the FAC system via the shear Alfven wave.

\footnotetext{
References

Amm, O., R. Fujii, K. Kauristie, A. Aikio, A. Yoshikawa, A. Ieda, and H. Vanhamäki (2011), A statistical investigation of the Cowling channel efficiency in the auroral zone, J. Geophys. Res. , 116, A02304, doi:10.1029/2010JA015988.

Fujii, R., O. Amm, A. Yoshikawa, A. Ieda, and H. Vanhamäki (2011), Reformulation and energy flow of the Cowling channel, J. Geophys. Res., 116, A02305, doi:10.1029/2010JA015989.

Yoshikawa, A., H. Nakata, A. Nakamizo, T. Uozumi, M. Itonaga, S. Fujita, K. Yumoto, and T. Tanaka (2010), Alfveniccoupling algorithm for global and dynamical magnetosphere-ionosphere coupled system, J. Geophys. Res., 115, A04211, doi:10.1029/2009JA014924.

Yoshikawa, A., A. Nakamizo, O. Amm, H. Vanhamäki, R. Fujii, Y.-M. Tanaka, T. Uozumi, K. Yumoto, and S. Ohtani (2011a), Self-consistent formulation for the evolution of ionospheric conductances at the ionospheric $E$ region within the M-I coupling scheme, J. Geophys. Res., 116, A09223, doi:10.1029/2011JA016449.

Yoshikawa, A., O. Amm, H. Vanhamäki, and R. Fujii (2011b), A self-consistent synthesis description of magnetosphereionosphere coupling and scale-dependent auroral process using shear Alfvén wave, J. Geophys. Res., 116, A08218, doi:10.1029/2011JA016460.
} 